\title{
The effect of past antibiotic exposure on diabetes risk
}

\author{
Ben Boursi ${ }^{1,2,3}$, Ronac Mamtani', Kevin Haynes ${ }^{1}$ and Yu-Xiao Yang ${ }^{1, *}$ \\ ${ }^{1}$ Perelman School of Medicine at the University of Pennsylvania, Philadelphia, Pennsylvania, USA, ${ }^{2}$ The Integrated \\ Cancer Prevention Center, Tel-Aviv Sourasky Medical Center, Tel-Aviv, Israel and ${ }^{3}$ Tel-Aviv University, Tel-Aviv, Israel \\ *(Y-X Yang is now at Division of Gastroenterology, Department of Medicine, Department of Epidemiology \\ and Biostatistics, Center for Clinical Epidemiology and Biostatistics, Perelman School of Medicine at the \\ University of Pennsylvania, 733 Blockley Hall, 423 Guardian Drive, Philadelphia, Pennsylvania 19104-6021, USA)
}

\author{
Correspondence \\ should be addressed \\ to Y-X Yang \\ Email \\ yangy@mail.med.upenn.edu
}

\begin{abstract}
Objective: Gut microbiota influence metabolic pathways related to the pathogenesis of obesity, insulin-resistance and diabetes. Antibiotic therapy can alter the microbiota, and is commonly used in western countries. We sought to evaluate whether past antibiotic exposure increases diabetes risk.

Research design and methods: We conducted a nested case-control study using a large population-based database from the UK. The cases were defined as those with incident diagnosis of diabetes. For every case, four eligible controls matched on age, sex, practice-site, and duration of follow-up before index-date were selected using incidence-density sampling. Exposure of interest was antibiotic therapy $>1$ year before index-date. Odds ratios (ORs) and $95 \% \mathrm{Cls}$ were estimated using conditional logistic regression. The risk was adjusted for BMI, smoking, last glucose level, and number of infections before index-date, as well as past medical history of coronary artery disease and hyperlipidaemia.

Results: The study included 208002 diabetic cases and 815576 matched controls. Exposure to a single antibiotic prescription was not associated with higher adjusted diabetes risk. Treatment with two to five antibiotic courses was associated with increase in diabetic risk for penicillin, cephalosporins, macrolides and quinolones with adjusted OR ranging from 1.08 (95\% Cl 1.05-1.11) for penicillin to $1.15(95 \% \mathrm{Cl} 1.08-1.23)$ for quinolones. The risk increased with the number of antibiotic courses and reached 1.37 (95\% Cl 1.19-1.58) for more than 5 courses of quinolones. There was no association between exposure to anti-virals and anti-fungals and diabetes risk.
\end{abstract}

Conclusions: Exposure to certain antibiotic groups increases diabetes risk.

\section{Introduction}

The composition and diversity of the gut microbiota affect pathways related to metabolism, inflammation and immunity, and are highly associated with human health and disease. Specific changes in the microbiota were described in a wide range of diseases from inflammatory bowel disease to atherosclerosis and cancer $(1,2,3,4)$.

In the past decades, obesity, insulin resistance and type 2 diabetes have become a worldwide epidemic (5). Recent studies have emphasised the role of human gut (c) 2015 European Society of Endocrinology Printed in Great Britain microbiota in the pathogenesis of those metabolic conditions, showing increased prevalence among individuals with altered microbiota and low bacterial diversity $(6,7)$. Additional studies demonstrated different microbiota composition in children with type 1 diabetes. Those works suggested difference in immune reactivity against certain bacterial strains in the pathogenesis of the disease as well as other autoimmune diseases $(8,9)$. 
Diet may alter the microbiota composition, influencing the body's ability to utilise energy from food. Changes in the relative abundance of Bacteroidetes and Firmicutes, the two dominant bacterial phyla in the human gut, have been described in obese subjects (6). Diets high in animal protein and animal fat were also associated with Bacteroides abundance, while Prevotella was associated with diets high in carbohydrates $(7,10,11)$. Similarly, in the $\mathrm{Ob} / \mathrm{Ob}$ mice model, there was reduced abundance of Bacteroidetes and increase in Firmicutes compared with lean mice. Those changes in microbiota composition were shown to increase the capacity to harvest energy from diet (12). Germ-free mice remain lean despite consuming a high-fat, sugar-rich diet (13), and compensate for the reduced energy harvest due to lack of microbiota by higher dietary intake (14). Moreover, the obese trait was shown to be transmissible by colonisation of germ-free mice with obese mice microbiota (15).

Prolonged inflammation was also associated with insulin resistance and diabetes. Several works suggest that gut micobiota is involved in chronic intestinal inflammation (5), increased gastro-intestinal permeability and metabolic endotoxaemia, contributing to the development of insulin resistance (16). An increase in the level of bacterial 16S rDNA in blood was associated with higher diabetes risk in humans (17). In addition, mice deficient in TLR5 or in components of the inflammasome were prone to metabolic syndrome and type 2 diabetes $(18,19)$.

Moreover, microbial products, such as short-chain fatty acids, affect metabolism (2). Those small molecules were also shown to regulate intestinal gluconeogenesis, thus influencing diabetes risk $(20,21)$.

Two recent studies have demonstrated change in gut microbiota in patients with type 2 diabetes. In those large metagenome-wide association studies from China and Europe, there was an increase in the gram negative Bacteroides species and certain Lactobacillus species among diabetic patients as well as increased expression of pro-inflammatory genes $(3,4)$.

Two additional clinical observations support the association between gut microbiota and diabetes risk. Following faecal transplantation from lean donors, insulin sensitivity of recipients with metabolic syndrome increased along with the levels of butyrate-producing intestinal microbiota (22). In post-gastric bypass individuals, diabetes can resolve even before any change in weight, possibly due to a shift in bacterial population with reduction in Firmicutes and increase in Bacteroides species.
Those changes may be caused by the surgical procedure itself or be the consequence of change in diet $(23,24)$.

Antibiotic therapy can alter the gut microbiota. In mice model, low dose penicillin disrupt the microbiota, alter metabolic genes and induce adiposity $(25,26)$. In both $\mathrm{Ob} / \mathrm{Ob}$ and diet-induced obese mice, antibiotic therapy maximally suppressing the numbers of aerobic and anaerobic bacteria improved glycaemic control independent of food intake and changed the hormonal, inflammatory and metabolic status of the host (27). Recent works in humans have shown that antibiotic exposure among infants during the first year of life was associated with an increased the risk of overweight and central adiposity later in life (28). However, it's unclear whether change in the microbiota is the cause or a consequence of insulin resistance and diabetes.

Since antibiotics are commonly used in western countries, the aim of the current study is to evaluate whether past antibiotic exposure is associated to diabetes risk.

\section{Subjects and methods}

\section{Study design}

We conducted a nested case-control study with incidence density sampling using the health improvement network (THIN), a large population-based database from the UK. This design is computationally more efficient than a cohort study, and produces odds ratios (ORs) that are unbiased estimates of incidence rate ratios (29). The study was approved by the Institutional Review Board at the University of Pennsylvania and by the Scientific Review Committee of THIN.

\section{Data source}

THIN is a large population-based electronic medical records database from the UK that contains comprehensive medical records of approximately ten million patients under the care of general practitioners in 570 practices. THIN includes information on patient demographics, socioeconomic status, medical diagnoses, laboratory results and drug prescriptions. Under the National Health Services, $98 \%$ of the UK population receives all forms of health care through their general practitioners, thus the demographic and geographic distributions of the THIN population are broadly representative of those of the general UK. Registration date is defined as the date when patients were first registered with a practice in THIN and Vision date is the date that a practice began using 
in-practice Vision Software which collects information for the THIN database (30). Data quality is monitored through routine analysis of the entered data $(31,32)$. The database has been previously used for pharmacoepidemiology studies, showing excellent quality of information $(33,34)$.

\section{Study cohort}

All people who received medical care from 1995 to 2013 from a THIN practitioner were eligible for inclusion. 1804170 (15.34\%) patients without acceptable medical records (i.e. patients with incomplete documentation or out of sequence date of birth, registration date, date of death, or date of exit from the database) were excluded. The follow-up started at the later of either the Vision date or 183 days after the date at which the patient registered with the general practitioner (35), and ended on the diabetes diagnosis date for those diagnosed with diabetes during the follow-up and on the earliest of date of death, transferring out of the database, or reach the end date of the database for controls.

\section{Case selection}

Cases were defined as all individuals in the cohort with at least one Read code (the standard clinical terminology system used in general practices in the UK) for diabetes mellitus during the follow-up period. Subjects who were diagnosed with the diabetes within the first 183 days after initiation of follow-up were excluded in order to avoid prevalent cases (35).

\section{Selection of controls}

Selection of the control group was based on incidencedensity sampling (29). The eligible control pool for each case consisted of all individuals without a diagnosis of diabetes at the date the case was diagnosed. For each case, up to four eligible controls were matched on age at index, sex, practice site, duration and calendar period of follow-up.

\section{Exposures and covariates}

The primary exposure of interest was any exposure more than 1 year before the index date to one of seven antibiotic groups commonly used in the outpatient setting: penicillins, cephalosporins, macrolides, tetracyclines, sulphonamides, quinolones and imidazole. The analysis was performed for each antibiotic class separately. We also assessed anti-viral and anti-fungal medications as potential negative controls. For each of the antibiotic classes, we evaluated the effect of number of antibiotic courses $(0,1,2-5$ and $>5$ courses). As potential confounders, we examined a comprehensive list of variables including obesity (BMI $>30$ ); smoking history (ever or never); medical co-morbidities including coronary artery disease and hyperlipidaemia treated with statins; number of previous urinary, skin and respiratory infections; and last glucose measure before or at diagnosis date. All covariates were measured before the index date.

\section{Statistical analyses}

The baseline characteristics of cases and controls were compared using $\chi^{2}$ test for categorical variables and $t$-test for continuous variables. The primary analysis was a conditional logistic regression to estimate ORs and 95\% CIs for the association between number of antibiotic courses for each antibiotic class and diabetes risk. The reference exposure group consisted of individuals without documented therapy with the specific antibiotic. The analysis was adjusted for all potential confounders. Bonferroni correction was performed to account for multiple comparisons due to assessment of nine treatment groups (seven antimicrobial groups as well as anti-viral and anti-fungal). The analysis was repeated only among individuals without urinary or skin infections before index date to minimise indication bias. For penicillins, due to the large number of prescriptions and high statistical power, we were able to repeat the analysis among individuals with last antibiotic prescription more than 5 years before diagnosis date. In this analysis, we did not adjust to last glucose value and the number of infections before index date as they occurred after exposure. In an additional sensitivity analysis, we evaluated the association between antibiotic exposure and auto-immune type 1 diabetes compared with type 2 disease that is associated with insulin resistance. The definition for type 1 diabetes included individuals with specific Read codes for the disease, individuals who initiated insulin therapy within 90 days of diagnosis and individuals below the age of 30 at the time of diagnosis. Furthermore, since glucose level might be part of the causal pathway between insulin resistance and diabetes, infection and antibiotic use we repeated the analysis without adjustment to this variable.

All analyses were performed using STATA 13 (Stata Corp., College Station, TX, USA). 


\section{Results}

The study population included 208002 diabetic cases and 815576 matched controls (Table 1). As expected, diabetic patients had elevated risk for high BMI, hyperlipidaemia and coronary artery disease, as part of the metabolic syndrome. In addition, cases had higher rate of infections before diagnosis date compared with controls, especially of the urinary tract (19.3\% vs $15.1 \%)$ and skin (17.8\% vs $12.4 \%)$. The most commonly prescribed antibiotic among the study population was penicillin, with 426159 subjects (41.6\%) receiving at least one prescription during followup period, followed by macrolides (150 996 subjects, 14.8\%) and TMP/SMX (120 494 subjects, 11.8\%).

The univariate analysis demonstrated increased diabetes risk with all antibiotic groups, reaching to an OR of 2.12 (95\% CI 2.08-2.17) for exposure to more than five antibiotic courses of penicillins (Table 2). In the multivariate analysis, a single antibiotic prescription more than 1 year before diagnosis date was not associated with higher adjusted diabetes risk in all antibiotic groups after applying the Bonferroni correction. The only exception was treatment with cephalosporins that was associated with a modest increase in diabetes risk (adjusted OR 1.09, 95\% CI 1.05-1.13). Treatment with two to five antibiotic courses was associated with increase in diabetes risk for penicillin, cephalosporins, macrolides and quinolones with adjusted OR ranging from 1.08 (95\% CI 1.05-1.11) for penicillin to 1.15 (95\% CI 1.08-1.23) for quinolones. The risk increased with the number of antibiotic courses and reached 1.37 (95\% CI 1.19-1.58) for more than five courses of quinolones. For two to five antibiotic courses, there was a modest increase in diabetes risk with TMP/SMX use (adjusted OR 1.1, 95\% CI 1.05-1.15). For more than five antibiotic courses, there was also an elevated risk with tetracyclines (adjusted OR 1.21, 95\% CI 1.09-1.33). There was no association between treatment with imidazole, anti-virals and anti-fungals and diabetes risk independent of the number of treatment courses (Table 2).

In a sensitivity analysis performed only among subjects without urinary tract or skin infections before index date, there was no change in results apart from lack of association between any number of TMP/SMX courses and diabetes risk (Table 3). In additionally, among subjects on last penicillin prescription more than 5 years before diagnosis with diabetes, the unadjusted ORs were 1.18 (95\% CI 1.15-1.21), 1.57 (95\% CI 1.43-1.74) and 1.87 (95\% CI 1.39-2.52) for $1,2-5$ and more than five antibiotic courses, respectively, all with $P<0.0001$. The adjusted ORs were 1.05 (95\% CI 1.02-1.08, $P=0.002), 1.23$ (95\% CI 1.1-1.37, $P<0.0001), 1.33$ (95\% CI 0.95-1.86, $P=0.09)$ respectively.

When only subjects with type 1 diabetes were analysed, there was an association only between exposure to more than five courses of penicillin or two to five courses of cephalosporin and disease risk (ORs 1.41 95\% CI 1.11-1.78 and 1.63 95\% CI 1.26-2.11 respectively). No association was observed between other antibiotic groups or number of antibiotic courses prescribed and diabetes risk (Table 4).

In an additional sensitivity analysis not adjusted to last glucose level before or at diagnosis, there was an even higher increase in diabetes risk. In this analysis, the risk was demonstrated with all antibiotic groups and with both type 1 and type 2 diabetes (data not shown). The risk for type 1 diabetes increased with the number of antibiotic courses from 1.09 (95\% CI 1.03-1.16) for a single course of penicillin to 1.67 (95\% CI 1.53-1.83) for more than five courses.

Table 1 Characteristics of cases and controls.

\begin{tabular}{lc}
\hline & Cases $(n=208002)$ \\
\hline Age (years, s.D.) & $60.5(16.0)$ \\
Sex (\% male, $n)$ & $53.3 \%(110831)$ \\
Follow-up duration (years, s.D.) & $5.5(3.9)$ \\
Obesity (BMI > 30) $(\%, n)$ & $40.6 \%(84415)$ \\
Ever smoking $\%, n)$ & $46.6 \%(96977)$ \\
Coronary artery disease (\%, $n)$ & $13.3 \%(27694)$ \\
Hyperlipidaemia Rx statins $(\%, n)$ & $22.1 \%(45905)$ \\
Urinary tract infection $(\%, n)$ & $19.3 \%(40051)$ \\
Skin infection $(\%, n)$ & $17.8 \%(37082)$ \\
Pneumonia (\%, $n)$ & $3.8 \%(7954)$ \\
Upper respiratory tract infection $(\%, n)$ & $16.9 \%(35225)$ \\
Last glucose before or at diagnosis & $9.1(3.8)$ \\
$\quad$ date (mmol/l, s.D.) & \\
\hline
\end{tabular}

\begin{tabular}{c}
\hline Controls $(n=815576)$ \\
\hline $60.2(16.0)$ \\
$53.3 \%(434574)$ \\
$5.5(3.9)$ \\
$15.1 \%(123300)$ \\
$36.4 \%(296843)$ \\
$7.0 \%(56895)$ \\
$13.5 \%(109683)$ \\
$15.1 \%(123329)$ \\
$12.4 \%(101027)$ \\
$2.7 \%(21727)$ \\
$14.1 \%(114990)$ \\
$5.3(1.0)$ \\
\end{tabular}

\begin{tabular}{c}
\hline Crude OR \\
\hline NA \\
NA \\
NA \\
$4.49(4.44-4.55)$ \\
$1.65(1.63-1.67)$ \\
$2.18(2.14-2.21)$ \\
$2.1(2.07-2.13)$ \\
$1.05(1.04-1.05)$ \\
$1.11(1.11-1.12)$ \\
$1.28(1.26-1.31)$ \\
$1.11(1.1-1.11)$ \\
$2.76(2.73-2.79)$ \\
\end{tabular}

www.eje-online.org 
Table 2 Diabetes risk as a function of the number of specific antibiotic courses.

\begin{tabular}{l}
$\begin{array}{l}\text { Antibiotic } \\
\text { type }\end{array}$ \\
\hline
\end{tabular}

Penicillins

2

Cephalosporines

Number of
antibiotic courses

0

1

$2-5$

$>5$

$$
\begin{gathered}
0 \\
1 \\
2-5 \\
>5
\end{gathered}
$$

Macrolides

.

\section{Quinolones}

8

$$
\text { (1) }
$$

Tetracyclines

$2-5$

Imidazole

$>$

Anti-fungals

$$
0
$$

Anti-fungals 2

Anti-virals

$$
\begin{gathered}
0 \\
1 \\
2-5 \\
>5
\end{gathered}
$$

\begin{tabular}{c}
\hline $\begin{array}{c}\text { Cases } \\
(n=208002)\end{array}$ \\
\hline $110539(53.14 \%)$ \\
$34344(16.51 \%)$ \\
$45356(21.81 \%)$ \\
$17763(8.54 \%)$
\end{tabular}

$181218(87.12 \%)$

$15497(7.45 \%)$

$9266(4.45 \%)$

$2021(0.97 \%)$

$170682(82.06 \%)$

$20535(9.87 \%)$

$13654(6.56 \%)$

3131 (1.51\%)

$192616(92.6 \%)$

$9803(4.71 \%)$

4675 (2.25\%)

$908(0.44 \%)$

$180047(86.56 \%)$

$16375(7.87 \%)$

9652 (4.64\%)

1928 (0.93\%)

$186719(89.77 \%)$

$12902(6.2 \%)$

6527 (3.14\%)

1854 (0.89\%)

$198482(95.42 \%)$ $7129(3.43 \%)$

$2202(1.06 \%)$

$189(0.09 \%)$

195344 (93.91\%) $6833(3.29 \%)$

4683 (2.25\%)

$1142(0.55 \%)$

$203035(97.61 \%)$

$4351(2.09 \%)$

465 (0.22\%)

$151(0.07 \%)$

\begin{tabular}{c}
$\begin{array}{c}\text { Controls } \\
(n=815576)\end{array}$ \\
\hline $486880(59.7 \%)$ \\
$133063(16.32 \%)$ \\
$150883(18.5 \%)$ \\
$44750(5.49 \%)$
\end{tabular}

$734860(90.1 \%)$

$49092(6.02 \%)$

$26889(3.3 \%)$

4735 (0.58\%)

$701900(86.06 \%)$

$67448(8.27 \%)$

38824 (4.76\%)

$7404(0.91 \%)$

770205 (94.44\%)

$30392(3.73 \%)$

12866 (1.58\%)

$2113(0.26 \%)$

723037 (88.65\%)

$56353(6.91 \%)$

30700 (3.76\%)

$5486(0.67 \%)$

745298 (91.38\%)

$42940(5.26 \%)$

$21068(2.58 \%)$

$6270(0.77 \%)$

$785703(96.34 \%)$

$23240(2.85 \%)$

$6245(0.77 \%)$

$388(0.05 \%)$

773351 (94.82\%)

$22275(2.73 \%)$

16216 (1.99\%)

3734 (0.46\%)

797785 (97.82\%)

15443 (1.89\%)

$1756(0.22 \%)$

$592(0.07 \%)$

Crude OR

1.24 (1.22-1.25)

$P<0.0001$

1.51 (1.49-1.53)

$P<0.0001$

$2.12(2.08-2.17)$

$P<0.0001$

References

1.34 (1.31-1.37)

$P<0.0001$

1.49 (1.46-1.53)

$P<0.0001$

1.89 (1.79-2.0)

$P<0.0001$

References

$1.3(1.28-1.32)$

$P<0.0001$

$1.52(1.49-1.55)$

$P<0.0001$

1.86 (1.78-1.94)

$P<0.0001$

References

1.31 (1.28-1.35)

$P<0.0001$

1.49 (1.44-1.54)

$P<0.0001$

1.78 (1.65-1.93)

$P<0.0001$

References

1.19 (1.17-1.22)

$P<0.0001$

1.31 (1.28-1.34)

$P<0.0001$

$1.48(1.41-1.56)$

$P<0.001$

References

$1.22(1.2-1.255)$

$P<0.0001$

1.27 (1.23-1.31)

$P<0.0001$

$1.21(1.15-1.28)$

$P<0.0001$

References

$1.23(1.2-1.27)$

$P<0.0001$

1.43 (1.36-1.51)

$P<0.0001$

1.97 (1.66-2.35)

$P<0.0001$

References

1.23 (1.19-1.26)

$P<0.0001$

$1.16(1.12-1.2)$

$P<0.0001$

1.23 (1.15-1.31)

$P<0.0001$

References

1.11 (1.07-1.15)

$P<0.0001$

1.05 (0.95-1.16)

$P=0.37$

$1.01(0.84-1.21)$

$P=0.91$
Adjusted OR ${ }^{a, b}$

References

1.02 (0.99-1.06)

$P=0.18$

$1.08(1.05-1.11)$

$P<0.0001$

1.23 (1.18-1.28)

$P<0.0001$

References

1.09 (1.05-1.13)

$P<0.0001$

1.11 (1.06-1.17)

$P<0.0001$

1.32 (1.19-1.46)

$P<0.0001$

References

1.05 (1.01-1.08)

$P=0.01$

1.11 (1.07-1.16)

$P<0.0001$

$1.32(1.22-1.43)$

$P<0.0001$

References

1.05 (1.0-1.1)

$P=0.05$

$1.15(1.08-1.23)$

$P<0.0001$

1.37 (1.19-1.58)

$P<0.0001$

References

0.98 (0.94-1.02)

$P=0.31$

$1.1(1.05-1.15)$

$P<0.0001$

1.08 (0.98-1.19)

$P=0.14$

References

1.06 (1.01-1.11)

$P=0.008$

$1.05(0.99-1.12)$

$P=0.07$

1.21 (1.09-1.33)

$P=0.001$

References

$1.06(1.0-1.12)$

$P=0.03$

1.06 (0.97-1.17)

$P=0.21$

$1.26(0.9-1.76)$

$P=0.18$

References

1.04 (0.98-1.09)

$P=0.22$

$1.05(0.99-1.12)$

$P=0.1$

$1.01(0.9-1.15)$

$P=0.82$

References

$0.99(0.93-1.06)$

$P=0.76$

$0.92(0.77-1.11)$

$P=0.4$

$0.95(0.69-1.31)$

$P=0.75$

${ }^{a}$ Adjusted for BMI, smoking, coronary artery disease, hyperlipidaemia treated with statins, last glucose level before index date, number of urinary tract, skin and respiratory infections before index date.

${ }^{b}$ After the Bonferroni correction, the $P$ value needed to show statistical significance was 0.006 . 
Table 3 Diabetes risk among subjects without previous urinary or skin infections, as a function of the number of specific antibiotic courses.

\begin{tabular}{|c|c|c|c|c|c|}
\hline Antibiotic type & $\begin{array}{c}\text { Number of } \\
\text { antibiotic courses }\end{array}$ & Cases $(n=139732)$ & Controls $(n=440587)$ & Crude OR & Adjusted OR ${ }^{a, b}$ \\
\hline \multirow[t]{3}{*}{ Penicillins } & $\begin{array}{l}0 \\
1\end{array}$ & $\begin{array}{l}80349(57.5 \%) \\
23702(16.96 \%)\end{array}$ & $\begin{array}{c}283591(64.37 \%) \\
70488(16.0 \%)\end{array}$ & $\begin{array}{l}\text { References } \\
1.25(1.23-1.28) \\
P<0.0001\end{array}$ & $\begin{array}{l}\text { References } \\
1.03(0.99-1.08) \\
P=0.13\end{array}$ \\
\hline & $2-5$ & $27607(19.76 \%)$ & $70447(15.99 \%)$ & $\begin{array}{l}1.52(1.49-1.54) \\
P<0.0001\end{array}$ & $\begin{array}{l}1.1(1.06-1.15) \\
P<0.0001\end{array}$ \\
\hline & $>5$ & $8074(5.78 \%)$ & $16061(3.65 \%)$ & $\begin{array}{l}2.03(1.96-2.09) \\
P<0.0001\end{array}$ & $\begin{array}{l}1.26(1.18-1.34) \\
P<0.0001\end{array}$ \\
\hline \multirow[t]{3}{*}{ Cephalosporines } & $\begin{array}{l}0 \\
1\end{array}$ & $\begin{array}{c}127493(91.24 \%) \\
8112(5.81 \%)\end{array}$ & $\begin{array}{c}412250(93.57 \%) \\
19448(4.41 \%)\end{array}$ & $\begin{array}{l}\text { References } \\
1.35(1.31-1.38) \\
P<0.0001\end{array}$ & $\begin{array}{l}\text { References } \\
1.13(1.06-1.2) \\
P<0.0001\end{array}$ \\
\hline & $2-5$ & $3663(2.62 \%)$ & $7985(1.81 \%)$ & $\begin{array}{l}1.49(1.43-1.55) \\
P<0.0001\end{array}$ & $\begin{array}{l}1.19(1.1-1.3) \\
P<0.0001\end{array}$ \\
\hline & $>5$ & $464(0.33 \%)$ & $904(0.21 \%)$ & $\begin{array}{l}1.68(1.49-1.88) \\
P<0.0001\end{array}$ & $\begin{array}{l}1.23(0.98-1.55) \\
P=0.08\end{array}$ \\
\hline \multirow[t]{3}{*}{ Macrolides } & $\begin{array}{l}0 \\
1\end{array}$ & $\begin{array}{c}118791(85.01 \%) \\
12300(8.8 \%)\end{array}$ & $\begin{array}{c}390337(88.59 \%) \\
31353(7.12 \%)\end{array}$ & $\begin{array}{l}\text { References } \\
1.3(1.27-1.33) \\
P<0.0001\end{array}$ & $\begin{array}{l}\text { References } \\
1.05(1.0-1.1) \\
P=0.08\end{array}$ \\
\hline & $2-5$ & $7274(5.21 \%)$ & $16314(3.7 \%)$ & $\begin{array}{l}1.48(1.44-1.53) \\
P<0.0001\end{array}$ & $\begin{array}{l}1.11(1.04-1.18) \\
P=0.001\end{array}$ \\
\hline & $>5$ & $1367(0.98 \%)$ & $2583(0.59 \%)$ & $\begin{array}{l}1.75(1.64-1.87) \\
P<0.0001\end{array}$ & $\begin{array}{l}1.45(1.27-1.67) \\
P<0.0001\end{array}$ \\
\hline \multirow[t]{3}{*}{ Quinolones } & $\begin{array}{l}0 \\
1\end{array}$ & $\begin{array}{c}132504(94.83 \%) \\
4963(3.55 \%)\end{array}$ & $\begin{array}{c}423175(96.05 \%) \\
12447(2.83 \%)\end{array}$ & $\begin{array}{l}\text { References } \\
1.27(1.23-1.32) \\
P<0.0001\end{array}$ & $\begin{array}{l}\text { References } \\
1.1(1.03-1.19) \\
P=0.007\end{array}$ \\
\hline & $2-5$ & $1968(1.41 \%)$ & $4447(1.01 \%)$ & $\begin{array}{l}1.42(1.34-1.5) \\
P<0.0001\end{array}$ & $\begin{array}{l}1.24(1.12-1.38) \\
P<0.0001\end{array}$ \\
\hline & $>5$ & $297(0.21 \%)$ & $518(0.12 \%)$ & $\begin{array}{l}1.85(1.6-2.14) \\
P<0.0001\end{array}$ & $\begin{array}{l}1.64(1.24-2.16) \\
P<0.0001\end{array}$ \\
\hline \multirow[t]{3}{*}{ TMP/SMX } & $\begin{array}{l}0 \\
1\end{array}$ & $\begin{array}{c}128308(91.82 \%) \\
7876(5.64 \%)\end{array}$ & $\begin{array}{c}410732(93.22 \%) \\
21019(4.77 \%)\end{array}$ & $\begin{array}{l}\text { References } \\
1.17(1.14-1.2) \\
P<0.0001\end{array}$ & $\begin{array}{l}\text { References } \\
0.97(0.91-1.03) \\
P=0.31\end{array}$ \\
\hline & $2-5$ & $3212(2.3 \%)$ & $7998(1.82 \%)$ & $\begin{array}{l}1.28(1.22-1.33) \\
P<0.0001\end{array}$ & $\begin{array}{l}1.12(1.03-1.22) \\
P=0.007\end{array}$ \\
\hline & $>5$ & $336(0.24 \%)$ & $838(0.19 \%)$ & $\begin{array}{l}1.29(1.14-1.47) \\
P<00.001\end{array}$ & $\begin{array}{l}1.1(0.86-1.4) \\
P=0\end{array}$ \\
\hline \multirow[t]{3}{*}{ Tetracyclines } & $\begin{array}{l}0 \\
1\end{array}$ & $\begin{array}{c}127458(91.22 \%) \\
7611(5.45 \%)\end{array}$ & $\begin{array}{c}408529(92.72 \%) \\
19855(4.51 \%)\end{array}$ & $\begin{array}{l}\text { References } \\
1.22(1.18-1.25) \\
P<0.0001\end{array}$ & $\begin{array}{l}\text { References } \\
1.08(1.01-1.15) \\
P=0.02\end{array}$ \\
\hline & $2-5$ & $3590(2.57 \%)$ & $9273(2.1 \%)$ & $\begin{array}{l}1.23(1.18-1.28) \\
P<0.0001\end{array}$ & $\begin{array}{l}1.04(0.95-1.13) \\
P=0.42\end{array}$ \\
\hline & $>5$ & $1073(0.77 \%)$ & $2930(0.67 \%)$ & $\begin{array}{l}1.18(1.1-1.26) \\
P<0.0001\end{array}$ & $\begin{array}{l}1.28(1.1-1.48) \\
P=0.001\end{array}$ \\
\hline \multirow[t]{3}{*}{ Imidazole } & $\begin{array}{l}0 \\
1\end{array}$ & $\begin{array}{c}134909(96.55 \%) \\
3782(2.71 \%)\end{array}$ & $\begin{array}{c}428520(97.26 \%) \\
9686(2.2 \%)\end{array}$ & $\begin{array}{l}\text { References } \\
1.2(1.16-1.25) \\
P<0.0001\end{array}$ & $\begin{array}{l}\text { References } \\
1.04(0.96-1.13) \\
P=0.33\end{array}$ \\
\hline & $2-5$ & $969(0.69 \%)$ & $2248(0.51 \%)$ & $\begin{array}{l}1.33(1.23-1.44) \\
P<0.0001\end{array}$ & $\begin{array}{l}1.05(0.9-1.23) \\
P=0.5\end{array}$ \\
\hline & $>5$ & $72(0.05 \%)$ & $133(0.03 \%)$ & $\begin{array}{l}1.71(1.28-2.29) \\
P<0.0001\end{array}$ & $\begin{array}{l}1.36(0.75-2.48) \\
P=0.31\end{array}$ \\
\hline \multirow[t]{3}{*}{ Anti-fungals } & $\begin{array}{l}0 \\
1\end{array}$ & $\begin{array}{c}137722(94.98 \%) \\
3855(2.76 \%)\end{array}$ & $\begin{array}{c}421447(95.66 \%) \\
10127(2.3 \%)\end{array}$ & $\begin{array}{l}\text { References } \\
1.21(1.16-1.25) \\
P<0.0001\end{array}$ & $\begin{array}{l}\text { References } \\
1.04(0.96-1.13) \\
P=0.34\end{array}$ \\
\hline & $2-5$ & $2552(1.83 \%)$ & $7311(1.66 \%)$ & $\begin{array}{l}1.11(1.06-1.16) \\
P<0.0001\end{array}$ & $\begin{array}{l}1.05(0.96-1.16) \\
P=0.27\end{array}$ \\
\hline & $>5$ & $603(0.43 \%)$ & $1702(0.39 \%)$ & $\begin{array}{l}1.13(1.02-1.24) \\
P=0.01\end{array}$ & $\begin{array}{l}0.98(0.82-1.18) \\
P=0.85\end{array}$ \\
\hline \multirow[t]{3}{*}{ Anti-virals } & $\begin{array}{l}0 \\
1\end{array}$ & $\begin{array}{c}136862(97.95 \%) \\
2514(1.8 \%)\end{array}$ & $\begin{array}{c}432631(98.19 \%) \\
6857(1.56 \%)\end{array}$ & $\begin{array}{l}\text { References } \\
1.11(1.06-1.17) \\
P<0.0001\end{array}$ & $\begin{array}{l}\text { References } \\
0.97(0.88-1.07) \\
P=0.52\end{array}$ \\
\hline & $2-5$ & $271(0.19 \%)$ & $847(0.19 \%)$ & $\begin{array}{l}1.02(0.88-1.17) \\
P=0.83\end{array}$ & $\begin{array}{l}0.86(0.66-1.13) \\
P=0.28\end{array}$ \\
\hline & $>5$ & $85(0.06 \%)$ & $252(0.06 \%)$ & $\begin{array}{l}1.08(0.84-1.38) \\
P=0.57\end{array}$ & $\begin{array}{l}1.05(0.67-1.66) \\
P=0.82\end{array}$ \\
\hline
\end{tabular}

${ }^{a}$ Adjusted for BMI, smoking, coronary artery disease, hyperlipidaemia treated with statins, last glucose level before index date, and number of respiratory infections before index date.

${ }^{b}$ After the Bonferroni correction, the $P$ value needed to show statistical significance was 0.006 . 
Table 4 Diabetes risk among subjects with type 1 diabetes as a function of the number of specific antibiotic courses.

\begin{tabular}{|c|c|c|c|c|c|}
\hline Antibiotic type & $\begin{array}{c}\text { Number of } \\
\text { antibiotic courses }\end{array}$ & Cases $(n=15$ 505) & Controls $(n=61418)$ & Crude OR & Adjusted OR ${ }^{a, b}$ \\
\hline \multirow[t]{3}{*}{ Penicillins } & $\begin{array}{l}0 \\
1\end{array}$ & $\begin{array}{l}8723(56.26 \%) \\
2333(15.05 \%)\end{array}$ & $\begin{array}{r}38025(61.91 \%) \\
9469(15.42 \%)\end{array}$ & $\begin{array}{l}\text { References } \\
1.19(1.13-1.26) \\
P<0.0001\end{array}$ & $\begin{array}{l}\text { References } \\
1.02(0.86-1.22) \\
P=0.8\end{array}$ \\
\hline & $2-5$ & $3186(20.55 \%)$ & $10810(17.6 \%)$ & $\begin{array}{l}1.54(1.46-1.62) \\
P<0.0001\end{array}$ & $\begin{array}{l}1.25(1.06-1.48) \\
P=0.007\end{array}$ \\
\hline & $>5$ & $1263(8.15 \%)$ & $3114(5.07 \%)$ & $\begin{array}{l}2.35(2.17-2.55) \\
P<0.0001\end{array}$ & $\begin{array}{l}1.41(1.11-1.78) \\
P=0.004\end{array}$ \\
\hline \multirow[t]{3}{*}{ Cephalosporines } & $\begin{array}{l}0 \\
1\end{array}$ & $\begin{array}{c}13953(89.99 \%) \\
933(6.02 \%)\end{array}$ & $\begin{array}{c}56900(92.64 \%) \\
2941(4.79 \%)\end{array}$ & $\begin{array}{l}\text { References } \\
1.38(1.27-1.49) \\
P<0.0001\end{array}$ & $\begin{array}{l}\text { References } \\
1.06(0.85-1.31) \\
P=0.626\end{array}$ \\
\hline & $2-5$ & $518(3.34 \%)$ & $1415(2.3 \%)$ & $\begin{array}{l}1.63(1.46-1.82) \\
P<0.0001\end{array}$ & $\begin{array}{l}1.63(1.26-2.11) \\
P<0.0001\end{array}$ \\
\hline & $>5$ & $101(0.65 \%)$ & $162(0.26 \%)$ & $\begin{array}{l}2.85(2.21-3.68) \\
P<0.0001\end{array}$ & $\begin{array}{l}1.99(1.09-3.66) \\
P=0.03\end{array}$ \\
\hline \multirow[t]{3}{*}{ Macrolides } & $\begin{array}{l}0 \\
1\end{array}$ & $\begin{array}{r}13071(84.3 \%) \\
1402(9.04 \%)\end{array}$ & $\begin{array}{r}54049(88.0 \%) \\
4499(7.33 \%)\end{array}$ & $\begin{array}{l}\text { References } \\
1.35(1.27-1.44) \\
P<0.0001\end{array}$ & $\begin{array}{l}\text { References } \\
1.1(0.91-1.32) \\
P=0.33\end{array}$ \\
\hline & $2-5$ & $837(5.4 \%)$ & $2455(4.0 \%)$ & $\begin{array}{l}1.51(1.39-1.65) \\
P<0.0001\end{array}$ & $\begin{array}{l}1.18(0.95-1.46) \\
P=0.14\end{array}$ \\
\hline & $>5$ & $195(1.26 \%)$ & $415(0.68 \%)$ & $\begin{array}{l}2.12(1.78-2.53) \\
P<0.0001\end{array}$ & $\begin{array}{l}1.55(1.01-2.38) \\
P=0.04\end{array}$ \\
\hline \multirow[t]{3}{*}{ Quinolones } & $\begin{array}{l}0 \\
1\end{array}$ & $\begin{array}{c}14858(95.83 \%) \\
415(2.68 \%)\end{array}$ & $\begin{array}{c}59782(97.34 \%) \\
1260(2.05 \%)\end{array}$ & $\begin{array}{l}\text { References } \\
1.36(1.21-1.52) \\
P<0.0001\end{array}$ & $\begin{array}{l}\text { References } \\
1.04(0.79-1.36) \\
P=0.78\end{array}$ \\
\hline & $2-5$ & $181(1.17 \%)$ & $338(0.55 \%)$ & $\begin{array}{l}2.24(1.86-2.7) \\
P<0.0001\end{array}$ & $\begin{array}{l}1.61(1.06-2.45) \\
P=0.03\end{array}$ \\
\hline & $>5$ & $51(0.33 \%)$ & $38(0.06 \%)$ & $\begin{array}{l}5.52(3.62-8.41) \\
P<0.0001\end{array}$ & $\begin{array}{l}2.1(0.86-5.21) \\
P=0.1\end{array}$ \\
\hline \multirow[t]{3}{*}{ TMP/SMX } & $\begin{array}{l}0 \\
1\end{array}$ & $\begin{array}{c}14050(90.62 \%) \\
914(5.89 \%)\end{array}$ & $\begin{array}{c}56716(92.34 \%) \\
3125(5.09 \%)\end{array}$ & $\begin{array}{l}\text { References } \\
1.21(1.12-1.31) \\
P<0.0001\end{array}$ & $\begin{array}{l}\text { References } \\
0.93(0.76-1.14) \\
P=0.47\end{array}$ \\
\hline & $2-5$ & $463(2.99 \%)$ & $1388(2.26 \%)$ & $\begin{array}{l}1.41(1.26-1.57) \\
P<0.0001\end{array}$ & $\begin{array}{l}1.12(0.86-1.44) \\
P=0.4\end{array}$ \\
\hline & $>5$ & $78(0.5 \%)$ & $189(0.31 \%)$ & $\begin{array}{l}1.74(1.33-2.28) \\
P<0.0001\end{array}$ & $\begin{array}{l}1.18(0.63-2.21) \\
P=0.6\end{array}$ \\
\hline \multirow[t]{3}{*}{ Tetracyclines } & $\begin{array}{l}0 \\
1\end{array}$ & $\begin{array}{c}14424(93.03 \%) \\
652(4.21 \%)\end{array}$ & $\begin{array}{c}57585(93.76 \%) \\
2171(3.53 \%)\end{array}$ & $\begin{array}{l}\text { References } \\
1.21(1.1-1.32) \\
P<0.0001\end{array}$ & $\begin{array}{l}\text { References } \\
1.18(0.94-1.49) \\
P=0.15\end{array}$ \\
\hline & $2-5$ & $319(2.06 \%)$ & $1194(1.94 \%)$ & $\begin{array}{l}1.08(0.95-1.23) \\
P=0.24\end{array}$ & $\begin{array}{l}0.95(0.71-1.29) \\
P=0.76\end{array}$ \\
\hline & $>5$ & $110(0.71 \%)$ & $468(0.76 \%)$ & $\begin{array}{l}0.95(0.77-1.17) \\
P=0.63\end{array}$ & $\begin{array}{l}1.08(0.65-1.78) \\
P=0.77\end{array}$ \\
\hline \multirow[t]{3}{*}{ Imidazole } & $\begin{array}{l}0 \\
1\end{array}$ & $\begin{array}{c}14934(96.32 \%) \\
385(2.48 \%)\end{array}$ & $\begin{array}{c}59655(97.13 \%) \\
1341(2.18 \%)\end{array}$ & $\begin{array}{l}\text { References } \\
1.17(1.04-1.32) \\
P=0.008\end{array}$ & $\begin{array}{l}\text { References } \\
0.88(0.67-1.15) \\
P=0.35\end{array}$ \\
\hline & $2-5$ & $176(1.14 \%)$ & $403(0.66 \%)$ & $\begin{array}{l}1.81(1.5-2.17) \\
P<0.0001\end{array}$ & $\begin{array}{l}1.19(0.82-1.73) \\
P=0.37\end{array}$ \\
\hline & $>5$ & $10(0.06 \%)$ & $19(0.03 \%)$ & $\begin{array}{l}2.22(1.03-4.78) \\
P=0.04\end{array}$ & $\begin{array}{l}1.75(0.37-8.2) \\
P=0.48\end{array}$ \\
\hline \multirow[t]{3}{*}{ Anti-fungals } & $\begin{array}{l}0 \\
1\end{array}$ & $\begin{array}{c}14725(94.97 \%) \\
463(2.99 \%)\end{array}$ & $\begin{array}{c}59072(96.18 \%) \\
1469(2.39 \%)\end{array}$ & $\begin{array}{l}\text { References } \\
1.28(1.15-1.43) \\
P<0.0001\end{array}$ & $\begin{array}{l}\text { References } \\
1.22(0.92-1.61) \\
P=0.17\end{array}$ \\
\hline & $2-5$ & $256(1.65 \%)$ & $764(1.24 \%)$ & $\begin{array}{l}1.37(1.19-1.59) \\
P<0.0001\end{array}$ & $\begin{array}{l}1.06(0.76-1.49) \\
P=0.72\end{array}$ \\
\hline & $>5$ & $61(0.39 \%)$ & $113(0.18 \%)$ & $\begin{array}{l}2.25(1.64-3.08) \\
P<0.0001\end{array}$ & $\begin{array}{l}1.29(0.64-2.61) \\
P=0.48\end{array}$ \\
\hline \multirow[t]{3}{*}{ Anti-virals } & $\begin{array}{l}0 \\
1\end{array}$ & $\begin{array}{c}15252(98.37 \%) \\
208(1.34 \%)\end{array}$ & $\begin{array}{c}60649(98.75 \%) \\
648(1.06 \%)\end{array}$ & $\begin{array}{l}\text { References } \\
1.27(1.08-1.49) \\
P=0.003\end{array}$ & $\begin{array}{l}\text { References } \\
1.27(0.89-1.82) \\
P=0.18\end{array}$ \\
\hline & $2-5$ & $32(0.21 \%)$ & $88(0.14 \%)$ & $\begin{array}{l}1.47(0.98-2.2) \\
P=0.07\end{array}$ & $\begin{array}{l}1.1(0.49-2.49) \\
P=0.82\end{array}$ \\
\hline & $>5$ & $13(0.08 \%)$ & $33(0.05 \%)$ & $\begin{array}{l}1.55(0.81-2.94) \\
P=0.18\end{array}$ & $\begin{array}{l}1.07(0.24-4.81) \\
P=0.93\end{array}$ \\
\hline
\end{tabular}

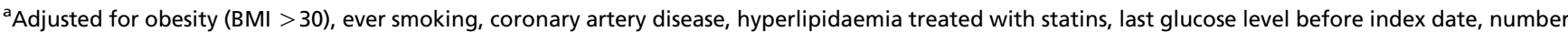
of urinary tract, skin and respiratory infections before index date.

${ }^{b}$ After the Bonferroni correction, the $P$ value needed to show statistical significance was 0.006 . 


\section{Discussion}

In this large population-based study, we showed an association between past antibiotic exposure and diabetes risk. This association was more pronounced with increased number of antibiotic courses prescribed. While the univariate analysis demonstrated a higher risk with exposure to all antibiotic groups and for both type 1 and type 2 diabetes, the adjusted analysis revealed an increase in risk only after exposure to more than one course of penicillin, cephalosporins, macrolides and quinolones, and showed almost no change in risk for participants with type 1 diabetes. The OR reached 1.15 (95\% CI 1.08-1.23, $P<0.0001)$ for two to five courses and 1.37 (95\% CI 1.19-1.58, $P<0.0001)$ for more than five courses of quinolones. There was no increase in risk with exposure to anti-viral or anti-fungal medications. There was no change in association when a stratified analysis only among subjects without previous urinary or skin infections before index date was performed.

Those results are consistent with previous studies in humans, showing microbial changes in patients with type 2 diabetes $(3,4)$ and increased risk of overweight among infants who were exposed to antibiotics during the first year of life (28). They are also consistent with mice models showing disruption of the microbiota, altered metabolic gene expression and induced adiposity following low dose antibiotic $(25,26)$. However, in models of obese mice, antibiotic administration with maximal suppression of the microbiota improved glycaemic control (27). One possible explanation is that certain bacteria are necessary in order to cause the phenotype of obesity and diabetes, and low dose antibiotics might increase the 'necessary pro-diabetes microbiota'. The fact that germfree mice remain lean even after consuming western diet supports the role of a 'pro-diabetes microbiota' (13).

The current study had more than one million subjects, of them 208002 diabetic patients, with a follow-up of up to 18 years. Only individuals who were diagnosed more than 6 months after initiation of follow-up were considered as cases in order to avoid prevalent cases. Over $40 \%$ of participants had at least one antibiotic prescription during the study duration. By matching cases and controls on duration and calendar period of follow-up as well as practice site, we avoided time-window bias and minimised confounding secondary to different clinical judgment regarding antibiotic prescription and type of antibiotic used.

In order to avoid possible protopathic bias (i.e. infections in the period before diagnosis could be the results of infections associated with undiagnosed diabetes), we analysed only antibiotic prescriptions given more than 1 year before diagnosis date. Adjustment to the number of previous urinary, skin and respiratory tract infections, as well as the sensitivity analysis among subjects without previous urinary and skin infections, known to be higher in diabetic patients, limited the possibility of confounding by indication. For penicillins, we were able to analyse the effect of antibiotic exposure more than 5 years before diabetes was diagnosed, allowing us to evaluate possible reverse causation in which the prediabetes state of insulin resistance increased infection risk and antibiotic use. The results for this sensitivity analysis were similar to the overall analysis pointing against possible overuse of antibiotics due to pre-diabetes state.

We further adjusted to obesity, hyperlipidaemia treated with statins, last glucose level before or at diagnosis and coronary artery disease. All those variables, together with diabetes, are part of the metabolic syndrome. Those parameters are associated with diabetes as well as with change in the microbiota and thus serve as possible confounders.

The current work had several limitations. Information regarding BMI was missing for $\sim 30 \%$ of the study population, because unlike prescription or medical diagnosis information in THIN, recording of BMI data was not mandated by the general practices. We analysed subjects with missing BMI information as a separate group and additionally performed complete-subject analysis which showed no change in results. Of note, obesity is the known risk factor for diabetes and metabolic syndrome, the percent of obese (BMI >30) participants in the current study were $\sim 22 \%$ compared with the reported $26 \%$ of the UK population in 2010 (HSCIC: Statistics on Obesity, Physical Activity and Diet - England, 2012).

The cases were defined according to at least one Read code for diabetes mellitus. Because many codes did not specify the type of diabetes that was diagnosed, in addition to specific type 1 diabetes codes, we included age $<30$ and initial insulin prescription within 90 days of diagnosis as additional definitions for the disease. The overall analysis was performed in patients with either type 1 or type 2 diabetes.

Our observational study did not include information regarding the microbiota composition of cases and controls and thus cannot infer causality. However, as change in the microbiota was previously demonstrated in humans in the entire spectrum of pathologies from obesity to insulin resistance and diabetes and as earlier studies showed antibiotic exposure as a risk factor for obesity $(3,4,28)$, our results may support a possible hypothesis that change in the gut microbiota secondary to antibiotic exposure increase diabetes risk. The need for 
recurrent antibiotic exposures in addition to the lack of known direct harmful effect of antibiotics on the $\beta$-cells of the pancreas is in concordance with such a hypothesis.

We had no information regarding whether the antibiotics prescribed were actually taken; however we expect that if compliance was actually lower it would have changed our results towards the null hypothesis. People may also use more than one type of antibiotic during follow-up, making it difficult to differentiate between the effects of different antibiotic groups. Our study results, showing differential effect between groups as well as compared with anti-viral and most anti-fungal medications, point against possible surveillance bias (i.e. people with infections are more likely to be diagnosed with diabetes). As we performed multiple comparison for exposure to various pre-defined antibiotic groups, we used the conservative Bonferonni correction in the final statistical analysis.

Importantly, although both unadjusted and adjusted analysis showed an association between antibiotic exposure and diabetes risk, the results differ in the magnitude of risk, the antibiotic groups associated with the risk and the effect on type 1 diabetes (increased risk in the univariate analysis vs almost no risk in the adjusted analysis). While the lack of association in type 1 diabetes might be explained by the different auto-immune pathogenesis of the disease, possibly viral induced $(36,37)$, we could not rule out over adjustment, decreasing the risk towards the null hypothesis, as an alternate explanation for our results. For this reason, we conducted a sensitivity analysis without adjustment for last glucose levels that might serve as part of a causal pathway between antibiotic exposure and diabetes risk. In this analysis, antibiotic exposure was associated with type 1 diabetes. These contrasting results prevent us from clear conclusions regarding type 1 diabetes and further studies are needed.

In summary, we demonstrated a higher adjusted risk for type 2 diabetes among individuals with recurrent exposures to penicillin, cephalosporins, macrolides and quinolones. There was no increase in adjusted risk for exposure to antiviral or anti-fungal medications. Despite the observational design and possibility of unmeasured confounding, the results are consistent with previous works that described altered microbiota in obese individuals as well as in subjects with insulin resistance and increase in obesity among individuals exposed to antibiotics during the first year of life. It is also consistent with animal models showing that the obese phenotype can be transformed by transplanting 'obesity-related bacteria' or induced by low dose antibiotic. Future studies in humans should focus on the changes in microbiota, which are caused by repeated antibiotic exposure; the effect of antibiotic exposure in different age groups; the genetic pathways that are affected by the microbiota; and whether antibiotic administration in diabetic patients might improve glycaemic control.

\section{Declaration of interest}

The authors declare that there is no conflict of interest that could be perceived as prejudicing the impartiality of the research reported.

\section{Funding}

The work was supported by the National Center for Research Resources and the National Center for Advancing Translational Sciences, National Institutes of Health, through Grant UL1TR000003. The content is solely the responsibility of the authors and does not necessarily represent the official views of the NIH.

Author contribution statement

Dr Y-X Yang and Dr B Boursi had full access to all of the data in the study and took responsibility for the integrity of the data and the accuracy of the data analysis. Y-X Yang, B Boursi, K Haynes and R Mamtani contributed to conception and design of the study; $Y$-X Yang and B Boursi acquired the data; Y-X Yang, B Boursi, K Haynes and R Mamtani contributed to analysis and interpretation of data, drafting the article or revising it critically for important intellectual content; and final approval of the version to be published.

\section{Acknowledgements}

The authors would also like to thank Nadir Arber MD MSc MHA, Head, The Integrated Cancer Prevention Center, Tel-Aviv University, Israel for reviewing the manuscript. Dr B Boursi would like to thank the Djerassi family for supporting his post-doctoral fellowship.

This work was performed in partial fulfilment of the requirements for a PhD degree of B Boursi, Sackler Faculty of Medicine, Tel-Aviv University, Israel

\section{References}

1 Turnbaugh PJ, Hamady M, Yatsunenko T, Cantarel BL, Duncan A, Ley RE, Sogin ML, Jones WJ, Roe BA, Affourtit JP et al. A core gut microbiome in obese and lean twins. Nature $2009457480-484$. (doi:10.1038/nature07540)

2 Albenberg LG \& Wu GD. Diet and the intestinal microbiome: associations, functions, and implications for health and disease. Gastroenterology 2014 146 1564-1572. (doi:10.1053/j.gastro.2014.01.058)

3 Qin J, Li Y, Cai Z, Li S, Zhu J, Zhang F, Liang S, Zhang W, Guan Y, Shen D et al. A metagenome-wide association study of gut microbiota in type 2 diabetes. Nature 2012490 55-60. (doi:10.1038/nature11450)

4 Karlsson FH, Tremaroli V, Nookaew I, Bergström G, Behre CJ, Fagerberg B, Nielsen J \& Bäckhed F. Gut metagenome in European women with normal, impaired and diabetic glucose control. Nature 2013498 99-103. (doi:10.1038/nature12198)

5 Johnson A \& Olefsky JM. The origins and drivers of insulin resistance. Cell 2013152 673-684. (doi:10.1016/j.cell.2013.01.041)

6 Tremaroli V \& Backhed F. Functional interactions between the gut microbiota and host metabolism. Nature 2012489 242-249. (doi:10.1038/nature11552) 
7 Le Chatelier E, Nielsen T, Qin J, Prifti E, Hildebrand F, Falony G, Almeida M, Arumugam M, Batto JM, Kennedy S et al. Richness of human gut microbiome correlates with metabolic markers. Nature 2013 500 541-546. (doi:10.1038/nature12506)

8 de Goffau MC, Fuentes S, van den Bogert B, Honkanen H, de Vos WM, Welling GW, Hyöty H \& Harmsen HJ. Aberrant gut microbiota composition at the onset of type 1 diabetes in young children. Diabetologia 201457 1569-1577. (doi:10.1007/s00125-014-3274-0)

9 Murri M, Leiva I, Gomez-Zumaquero JM, Tinahones FJ, Cardona F, Soriguer F \& Queipo-Ortuño MI. Gut microbiota in children with type 1 diabetes differs from that in healthy children: a case-control study. BMC Medicine 201311 46. (doi:10.1186/1741-7015-11-46)

10 Wu GD, Chen J, Hoffmann C, Bittinger K, Chen YY, Keilbaugh SA, Bewtra M, Knights D, Walters WA, Knight R et al. Linking long-term dietary patterns with gut microbial enterotypes. Science 2011334 105-108. (doi:10.1126/science.1208344)

11 De Filippo C, Cavalieri D, Di Paola M, Ramazzotti M, Poullet JB, Massart S, Collini S, Pieraccini G \& Lionetti P. Impact of diet in shaping gut microbiota revealed by a comparative study in children from Europe and rural Africa. PNAS 2010107 14691-14696. (doi:10.1073/ pnas.1005963107)

12 Ley RE, Bäckhed F, Turnbaugh P, Lozupone CA, Knight RD \& Gordon JI. Obesity alters gut microbial ecology. PNAS 2005102 11070-11075. (doi:10.1073/pnas.0504978102)

13 Bäckhed F, Manchester JK, Semenkovich CF \& Gordon JI. Mechanisms underlying the resistance to diet-induced obesity in germ-free mice. PNAS 2007104 979-984.

14 Wostmann BS, Larkin C, Moriarty A \& Bruckner-Kardoss E. Dietary intake, energy metabolism, and excretory losses of adult male germfree Wistar rats. Laboratory Animal Science 198333 46-50.

15 Turnbaugh PJ, Ley RE, Mahowald MA, Magrini V, Mardis ER \& Gordon JI. An obesity-associated gut microbiome with increased capacity for energy harvest. Nature $2006 \mathbf{4 4 4}$ 1027-1031. (doi:10.1038/nature05414)

16 Cani PD, Amar J, Iglesias MA, Poggi M, Knauf C, Bastelica D, Neyrinck AM, Fava F, Tuohy KM, Chabo C et al. Metabolic endotoxemia initiates obesity and insulin resistance. Diabetes 200756 1761-1772. (doi:10.2337/db06-1491)

17 Amar J, Serino M, Lange C, Chabo C, Iacovoni J, Mondot S, Lepage P, Klopp C, Mariette J, Bouchez $\mathrm{O}$ et al. Involvement of tissue bacteria in the onset of diabetes in humans: evidence for a concept. Diabetologia 201154 3055-3061. (doi:10.1007/s00125-011-2329-8)

18 Vijay-Kumar M, Aitken JD, Carvalho FA, Cullender TC, Mwangi S, Srinivasan S, Sitaraman SV, Knight R, Ley RE \& Gewirtz AT. Metabolic syndrome and altered gut microbiota in mice lacking Toll-like receptor 5. Science 2010328 228-231. (doi:10.1126/science.1179721)

19 Henao-Mejia J, Elinav E, Jin C, Hao L, Mehal WZ, Strowig T, Thaiss CA, Kau AL, Eisenbarth SC, Jurczak MJ et al. Inflammasome-mediated dysbiosis regulates progression of NAFLD and obesity. Nature 2012482 179-185. (doi:10.1038/nature10809)

20 De Vadder F, Kovatcheva-Datchary P, Goncalves D, Vinera J, Zitoun C, Duchampt A, Bäckhed F \& Mithieux G. Microbiota-generated metabolites promote metabolic benefits via gut-brain neural circuits. Cell 2014156 84-96. (doi:10.1016/j.cell.2013.12.016)

21 Tilg H \& Moschen AR. Microbiota and diabetes: an evolving relationship. Gut 201463 1513-1521. (doi:10.1136/gutjnl-2014-306928)

22 Vrieze A, Van Nood E, Holleman F, Salojärvi J, Kootte RS, Bartelsman JF, Dallinga-Thie GM, Ackermans MT, Serlie MJ, Oozeer R et al. Transfer of intestinal microbiota from lean donors increases insulin sensitivity in individuals with metabolic syndrome. Gastroenterology 2012143 913-916. (doi:10.1053/j.gastro.2012.06.031)
23 Furet JP, Kong LC, Tap J, Poitou C, Basdevant A, Bouillot JL, Mariat D, Corthier G, Doré J, Henegar C et al. Differential adaptation of human gut microbiota to bariatric surgery-induced weight loss: links with metabolic and low-grade inflammation markers. Diabetes 201059 3049-3057. (doi:10.2337/db10-0253)

24 Zhang H, DiBaise JK, Zuccolo A, Kudrna D, Braidotti M, Yu Y, Parameswaran P, Crowell MD, Wing R, Rittmann BE et al. Human gut microbiota in obesity and after gastric bypass. PNAS $2009 \mathbf{1 0 6}$ 2365-2370. (doi:10.1073/pnas.0812600106)

25 Cox LM, Yamanishi S, Sohn J, Alekseyenko AV, Leung JM, Cho I, Kim SG, Li H, Gao Z, Mahana D et al. Altering the intestinal microbiota during a critical developmental window has lasting metabolic consequences. Cell 2014158 705-721. (doi:10.1016/j.cell.2014.05.052)

26 Cho I, Yamanishi S, Cox L, Methé BA, Zavadil J, Li K, Gao Z, Mahana D, Raju K, Teitler I et al. Antibiotics in early life alter the murine colonic microbiome and adiposity. Nature 2012488 621-626. (doi:10.1038/ nature11400)

27 Membrez M, Blancher F, Jaquet M, Bibiloni R, Cani PD, Burcelin RG, Corthesy I, Macé K \& Chou CJ. Gut microbiota modulation with norfloxacin and ampicillin enhances glucose tolerance in mice. FASEB Journal 200822 2416-2426. (doi:10.1096/fj.07-102723)

28 Azad MB, Bridgman SL, Becker AB \& Kozyrskyj AL. Infant antibiotic exposure and the development of childhood overweight and central adiposity. International Journal of Obesity 201438 1290-1298. (doi:10.1038/ijo.2014.119)

29 Lubin JH \& Gail MH. Biased selection of controls for case-control analysis of cohort studies. Biometrics $1984 \mathbf{4 0} 63-75$. (doi:10.2307/2530744)

30 Maguire A, Blak BT \& Thompson M. The importance of defining periods of complete mortality reporting for research using automated data from primary care. Pharmacoepidemiology and Drug Safety 200918 76-83. (doi:10.1002/pds.1688)

31 Bourke A, Dattani H \& Robinson M. Feasibility study and methodology to create a quality-evaluated database of primary care data. Informatics in Primary Care 200412 171-177. (doi:10.14236/jhi.v12i3.124)

32 Lewis JD, Schinnar R, Bilker WB, Wang X \& Strom BL. Validation studies of the health improvement network (THIN) database for pharmacoepidemiology research. Pharmacoepidemiology and Drug Safety 200716 393-401. (doi:10.1002/pds.1335)

33 Hollowell J. The general practice research database: quality of morbidity data. Population Trends, 1997 36-40.

34 Blak BT, Thompson M, Dattani H \& Bourke A. Generalisability of The Health Improvement Network (THIN) database: demographics, chronic disease prevalence and mortality rates. Informatics in Primary Care 2011 19 251-255.

35 Lewis JD, Bilker WB, Weinstein RB \& Strom BL. The relationship between time since registration and measured incidence rates in the general practice research database. Pharmacoepidemiology and Drug Safety 200514 443-451. (doi:10.1002/pds.1115)

36 Laitinen OH, Honkanen H, Pakkanen O, Oikarinen S, Hankaniemi MM, Huhtala $\mathrm{H}$, Ruokoranta T, Lecouturier V, André P, Harju R et al. Coxsackievirus b1 is associated with induction of $\beta$-cell autoimmunity that portends type 1 diabetes. Diabetes 201463 446-455. (doi:10.2337/ db13-0619)

37 Oikarinen S, Tauriainen S, Hober D, Lucas B, Vazeou A, SioofyKhojine A, Bozas E, Muir P, Honkanen H, Ilonen J et al. Virus antibody survey in different European populations indicates risk association between Coxsackievirus b1 and type 1 diabetes. Diabetes 201463 655-662. (doi:10.2337/db13-0620)
Received 30 December 2014

Revised version received 22 February 2015

Accepted 27 February 2015 